\title{
Expected Values of Aggregation Operators on Cubic Triangular Fuzzy Number and Its Application to Multi-Criteria Decision Making Problems
}

\author{
Aliya Fahmi ${ }^{1}$, Saleem Abdullah ${ }^{2}$, Fazli Amin ${ }^{1}$, Asad Ali ${ }^{1}$, Khaista Rahman ${ }^{1}$ \\ ${ }^{1}$ Department of Mathematics, Hazara University, Mansehra, Pakistan \\ ${ }^{2}$ Department of Mathematics, Abdul Wali Khan University, Mardan, Pakistan \\ Email address: \\ aliyafahmi@gmail.com(A.Fahmi), Saleemabdullah81@yahoo.com(S. Abdullah), fazliamin@hu.edu.pk(F. Amin), \\ asad_maths@hu.edu.pk(A.Ali),khaista355@yahoo.com(K. Rahman)
}

\section{To cite this article:}

Aliya Fahmi, Saleem Abdullah, Fazli Amin, Asad Ali, Khaista Rahman. Expected Values of Aggregation Operators on Cubic Triangular Fuzzy Number and Its Application to Multi-Criteria Decision Making Problems. Engineering Mathematics. Vol. 2, No. 1, 2018 , pp. 1-11. doi: 10.11648/j.engmath.20180201.11

Received: December 26, 2017; Accepted: March 16, 2018; Published: May 31, 2018

\begin{abstract}
In this paper, we define triangular cubic fuzzy numbers and their operational laws. Originated on these operational laws, approximately aggregation operators, with triangular cubic fuzzy weighted arithmetic averaging operator and weighted geometric averaging operator are suggested. Expected values, score function and accuracy function of triangular cubic fuzzy numbers are defined. Founded on these, an amicable of triangular cubic fuzzy multi-criteria decision making method is proposed. By these aggregation operators, criteria values are aggregated and integrated triangular cubic fuzzy numbers of alternatives are conquered. By relating score function and accuracy function values of integrated fuzzy numbers, a positioning of the entire option set can be accomplished. An example is given to appear the achievability and convenience of the process.
\end{abstract}

Keywords: Triangular Cubic Fuzzy Numbers, Aggregation Operators, Multi-Criteria Decision Making

\section{Introduction}

There are a little opinion multi-criteria decision making (MCDM) problems in extensive sunshine commercial aspects. At present, the technique for MCDM with solid criteria's weight coefficients and criteria's measures ingest survived judiciously unlimited. Continually advanced the fuzzy set assumption was offered and used to yield carefulness of MCDM issues by Zadeh, fuzzy multi-criteria decision-making problems issues consume fixed active immense contemplation $[14,26]$. The fuzz in MCDM has the superior person achieve since the element that of the suggestion of fuzzy assortment [14]. There are a insufficient examinations at the performances for multi-criteria choicemaking issues, in which the criteria's weight coefficients are undisputable and the criteria's appearances are assured or are fuzzy numbers in $[9,11,23]$ and there are furthermore selected estimations on multi-criteria decision making or multi-criteria group decision making in $[26,24]$, where the problem coefficients are insufficient and the criteria's appearances are fuzzy numbers. The standards' fuzzy numbers and the criteria's weight coefficients are collected to be fuzzy numbers utilizing an equaled likely in those criticisms and at that point the standing or confederacy of regulations may be completed by contrasting and the fuzzy numbers. However, fuzzy numbers are used to attractiveness the fuzziness just through club grade. Not at all corresponding from fuzzy set, there's another parameter: non membership diploma in intuitionistic fuzzy set, that's applied to illustrate and individual the fuzzy pith of the goal global all of the greater expertly [10]. In this way, there are a insufficient views on it, be that as it may, the examinations are serious on their letterings, operations, family members and so on $[12,16]$ and there are uncommon studies on multicriteria intuitionistic fuzzy selection-making. Multi-criteria decision-making troubles, wherein the standards' weight coefficients and the standards' structures are both intuitionistic fuzzy sets are studied in [15] and the estimating procedures to take attention of the problems are proposed.

For multi-criteria selection-making problems, in which the substantial on standards' weight coefficients is disjointed and the standards' features are intuitionistic fuzzy components, 
the TOPSIS system and the VIKOR method are suggested in [20]. An evidential enquiring approach for MCDM in opinion of intuitionistic fuzzy sets is suggested in [19]. Intermission emolument intuitionistic fuzzy set is the evolution of intuitionistic fuzzy set, the club grade and the non-club diploma in interval intuitionistic fuzzy set are touched out to program language period morals from reliable numbers, the multi-criteria selection-making dilemmas founded completely on interval intuitionistic fuzzy set are deliberate in $[21,25]$ and increasing yearning approaches are suggested. In any case, intuitionistic fuzzy sets and interim intuitionistic fuzzy parts are similarly the proportional as fuzzy units, the grounds of which are distinct sets and intuitionistic fuzzy units are exploited to description the grade to which the foundation does or does now not have a domicile with some fuzzy ideas. The intuitionistic triangular fuzzy numbers and their operations are considered in [17], which are besides exploited as a part of guilt tree examination. Intuitionistic trapezoidal fuzzy numbers are existing in [22], which are the covering of intuitionistic triangular fuzzy numbers.

The indication of cubic set is a supposition of intuitionistic fuzzy sets, in which there are portrayals, one is utilized for the extent of matriculation and different is pragmatic for the level of non-participation. The participation proficiency is hold in the method of draw, at the same time as nonenrollment is difficulty the regular fuzzy set (Jun et al., 2010).

Fahmi et al. [2] developed the hamming distance for triangular cubic fuzzy number and weighted averaging operator. Fahmi et al. [3] proposed the cubic TOPSIS method and grey relational analysis set. Fahmi et al. [4] defined the triangular cubic fuzzy number and operational laws. The authors developed the triangular cubic fuzzy hybrid aggregation (TCFHA) administrator to total all individual fuzzy choice structure provide by the decision makers into the aggregate cubic fuzzy decision matrix. Amin et al. [1] defined the generalized triangular cubic linguistic hesitant fuzzy weighted geometric (GTCHFWG) operator, generalized triangular cubic linguistic hesitant fuzzy ordered weighted average (GTCLHFOWA) operator, generalized triangular cubic linguistic hesitant fuzzy ordered weighted geometric (GTCLHFOWG) operator, generalized triangular cubic linguistic hesitant fuzzy hybrid averag-ing (GTCLHFHA) operator and generalized triangular cubic linguistic hesitant fuzzy hybrid geometric (GTCLHFHG) operator. Fahmi et al. [6] developed Trapezoidal linguistic cubic hesitant fuzzy TOPSIS method to solve the MCDM method based on trapezoidal linguistic cubic hesitant fuzzy TOPSIS method. Fahmi et al. [5] define aggregation operators for triangular cubic linguistic hesitant fuzzy sets which include cubic linguistic fuzzy (geometric) operator, triangular cubic linguistic hesitant fuzzy weighted geometric (TCLHFWG) operator, triangular cubic linguistic hesitant fuzzy ordered weighted geometric (TCHFOWG) operator and triangular cubic linguistic hesitant fuzzy hybrid geometric (TCLHFHG) operator. Fahmi et al. [7] defined the trapezoidal cubic fuzzyy weighted arithmetic averaging operator and weighted geometric averaging operator. Expected values, score function, and accuracy function of trapezoidal cubic fuzzy numbers are defined. Fahmi et al. [8] developed three arithmetic averaging operators, that is trapezoidal cubic fuzzy Einstein weighted averaging (TrCFEWA) operator, trapezoidal cubic fuzzy Einstein ordered weighted averaging (TrCFEOWA) operator and trapezoidal cubic fuzzy Einstein hybrid weighted averaging (TrCFEHWA) operator, for aggregating trapezoidal cubic fuzzy information.

In this article, a few aggregation operators of triangular cubic fuzzy numbers are described, the expected values, the score function and accuracy function of triangular cubic fuzzy numbers are defined and a simple ordering method of triangular cubic fuzzy numbers is proposed and used in multi-criteria decision making based on the score function and the accuracy function.

In this paper: In section 2, we define basic concept of fuzzy set and cubic set. In section 3, we define the idea of triangular cubic fuzzy number and operational laws. In section 4, we define the concept of aggregation operators on triangular cubic fuzzy numbers. In section 5 , we define the Expected values of cubic triangular fuzzy numbers and comparison between them. In section 6, we define Multicriteria decision making method based on cubic triangular fuzzy numbers. In section 7 , an expressive example is given to check the information. In section 8 , we give a conclusion.

\section{Preliminaries}

In this section, we initiated some basic define results about cubic set. Triangular cubic fuzzy number, Aggregation operators on cubic triangular fuzzy numbers and Expected values of cubic triangular fuzzy numbers and comparison between them, Multi-criteria decision making method based on cubic triangular fuzzy numbers.

Definition [2]. Give $\mathrm{H}$ a chance to be a universe of talk. The possibility of fuzzy set was speak to by Zadeh and characterized as taking after; $J=\left\{h, \tilde{\Omega}_{J}(h) \mid h \in H\right\}$. A fuzzy set in a set $\mathrm{H}$ is characterized $\tilde{\Omega}_{J}: H \rightarrow I, \arcsin \theta$ is a membership function, $\tilde{\Omega}_{J}(h)$ meant the degree of membership of the element $h$ to the set $H$, where $I=[0,1]$. The collection of all fuzzy subsets of $H$ is denoted by $I^{H}$. Characterize a connection on $I^{H}$ as takes after:

$$
\left(\forall \tilde{\Omega}, \eta \in I^{H}\right)(\tilde{\Omega} \leq \eta \Leftrightarrow(\forall h \in H)(\tilde{\Omega}(h) \leq \eta(h))) .
$$

Definition [10] Let $H$ be a non-empty set. A cubic set $F=(\tilde{\vartheta}, \tilde{\lambda})$ in $H$ is said to be an internal cubic set if $\tilde{\vartheta}^{-}(\tilde{h}) \leq \tilde{\lambda}(\tilde{h}) \leq \tilde{\vartheta}^{+}(\tilde{h})$ for all $\tilde{h} \in H$

Definition [10] Let $H$ be a non-empty set. A cubic set $F=(\tilde{\vartheta}, \tilde{\lambda})$ on $H$ is said to be an external cubic set if $\tilde{\lambda}(\tilde{h}) \notin\left(\tilde{\vartheta}^{-}(\tilde{h}), \tilde{\vartheta}^{+}(\tilde{h})\right)$ for all $\tilde{h} \in H$. 


\section{Cubic Fuzzy Numbers and Cubic Triangular Fuzzy Numbers}

Definition The Cubic fuzzy set on $H$ is a set $J=\left\{h,\left\langle\left[\Gamma_{j}^{-}(h), \Gamma_{j}^{+}(h)\right], \eta_{j}(h)\right\rangle: h \in H\right\} \quad$ where $\Gamma_{J}$ and $\eta_{j}$ are membership and non-membership function, respectively

$$
\begin{gathered}
\Gamma_{J}^{-}(h): h \rightarrow[0,1], h \in H \rightarrow \Gamma_{J}^{+}(h) \in[0,1] \\
\Gamma_{J}^{+}(h): h \rightarrow[0,1], h \in H \rightarrow \Gamma_{J}^{+}(h) \in[0,1] \\
\eta_{J}(h): h \rightarrow[0,1], h \in H \rightarrow \eta_{J}(h) \in[0,1]
\end{gathered}
$$

Definition The Cubic fuzzy set on $H$ is a set
$J=\left\{h,\left\langle\left[\Gamma_{j}^{-}(h), \Gamma_{j}^{+}(h)\right], \eta_{J}(h)\right\rangle: h \in H\right\}$ is called CF-normal, if there exist at least two points $h_{0}, h_{1} \in H$ such that $\Gamma^{-}\left(h_{0}\right)=[1,1], \Gamma^{+}\left(h_{0}\right)=[1,1]$ and $\eta\left(h_{1}\right)=1$. It is without difficulty seen that given cubic fuzzy set $J$ is CF-normal if there's as a minimum one point that genuinely belongs to $J$ and at the least one factor which does no longer belong to $J$.

Definition The Cubic fuzzy set

$$
J=\left\{h,\left\langle\left[\Gamma_{J}^{-}(h), \Gamma_{J}^{+}(h)\right], \eta_{J}(h)\right\rangle: h \in H\right\}
$$

of the real line is called CF-convex, if $\forall h_{1}, h_{2} \in H, \forall \lambda \in[0,1]$

$$
\left[\Gamma_{j}^{-}, \Gamma_{j}^{+}\right]\left(\lambda h_{1}+(1-\lambda) h_{2}\right) \geq\left[\Gamma_{j}^{-}, \Gamma_{j}^{+}\right]\left(h_{1}\right) \wedge\left[\Gamma_{j}^{-}, \Gamma_{j}^{+}\right]\left(h_{2}\right) ; \eta_{J}(h)\left(\lambda h_{1}+(1-\lambda) h_{2}\right) \geq \eta_{J}\left(h_{1}\right) \wedge \eta_{J}\left(h_{2}\right)
$$

Thus $J$ is CF-convex if its membership function is fuzzy convex and its nonmember ship function is fuzzy concave. Definition The Cubic fuzzy set

$$
J=\left\{h,\left\langle\left[\Gamma_{j}^{-}(h), \Gamma_{j}^{+}(h)\right], \eta_{J}(h)\right\rangle: h \in H\right\}
$$

of the real line is called cubic fuzzy number $(\mathrm{CFN})$ if

a) $J$ is CF-normal,

b) $J$ is CF-convex,

(c) $\left[\Gamma_{j}^{-}(h), \Gamma_{j}^{+}(h)\right]$ Are upper fuzzy semi continuous and lower fuzzy semi-continuous, $\eta_{J}(h)$ is fuzzy semi continuous,

(d) $J=\left\{h \in H \quad \mid \eta_{J}(h)<1\right\}$ is bounded.

Definition Let $\tilde{b}$ be the cubic triangular fuzzy number on the set of real numbers, its ivtfs

Characterized as:

$$
\mu_{\widetilde{b}}(x)=\left\{\begin{array}{lr}
f_{\widetilde{b}}^{L}(x) & \ddot{a} \leq x<\ddot{b} \\
f_{\widetilde{b}}^{R}(x), & \ddot{b} \leq x<\ddot{c} \\
0 & \text { otherwise }
\end{array}\right.
$$

and triangular fuzzy set

$$
v_{\widetilde{b}}(h)=\left\{\begin{array}{c}
g_{\widetilde{b}}^{L}(x), \\
g_{\widetilde{b}}^{L}(x) \\
0
\end{array}\right.
$$

$$
\begin{aligned}
& \ddot{a}_{1} \leq h<\ddot{b}_{1} \\
& \ddot{b}_{1}<h \leq \ddot{c}_{1} \\
& \text { otherwise }
\end{aligned}
$$

The cubic fuzzy number is meant as $\left.\widetilde{b}=\left\langle\left[\left((\ddot{a}, \ddot{b}, \ddot{c}) ; \mu_{\ddot{a}}^{-}, \mu_{\ddot{a}}^{+}\right)\right], v_{\ddot{a}}\right)\right\rangle$. Unique in relation to fuzzy numbers, cubic fuzzy numbers have another parameter: triangular fuzzy set, that's utilized to explicit the volume to which the selection makers suppose that the element does now not belong to $\left((\ddot{a}, \ddot{b}, \ddot{c}, d) ; v_{\ddot{a}}\right)$. Whenever $\mu_{\ddot{a}}^{-}(x)=[1,1]$ $\mu_{\ddot{a}}^{+}(x)=[1,1], \quad v_{\ddot{a}}=0$, is known as everyday cubic fuzzy number to be unique triangular cubic fuzzy number.

Definition

$$
f_{\tilde{b}}^{L}(x)=\frac{x-\ddot{a}}{\ddot{b}-\ddot{a}}\left[\mu_{\ddot{a}}^{-}, \mu_{\ddot{a}}^{+}\right], f_{\tilde{b}}^{R}(x)=\frac{\ddot{c}-x}{\ddot{c}-\ddot{b}}\left[\mu_{\tilde{b}}^{\bar{b}}, \mu_{\tilde{b}}^{+}\right], g_{\tilde{b}}^{L}(x)=\frac{\left(\ddot{b}_{1}-x\right) v_{\ddot{a}}}{\ddot{b}_{1}-\ddot{a}_{1}}, g_{\tilde{b}}^{R}(x)=\frac{(\ddot{c}-x) v_{\tilde{b}}}{\ddot{c}_{1}-\ddot{b}_{1}},
$$

the cubic fuzzy number is known as cubic triangular cubic fuzzy number.

Definition Let $\quad \tilde{b}_{1}=\left\langle\left[\left(\left(\ddot{a}_{1}, \ddot{b}_{1}, \ddot{c}_{1}\right) ;\left[\mu_{\ddot{a}_{1}}^{-}, \mu_{\ddot{a}_{1}}^{+}\right)\right] v_{\ddot{a}_{1}}\right)\right\rangle \quad$ and $\left.\widetilde{b}_{2}=\left\langle\left[\left(\left(\ddot{a}_{2}, \ddot{b}_{2}, \ddot{c}_{2}\right) ; \mu_{\ddot{a}_{2}}^{-}, \mu_{\ddot{a}_{2}}^{+}\right)\right], v_{\ddot{a}_{2}}\right)\right\rangle$ be two triangular cubic fuzzy numbers, then

(1):

$$
\begin{aligned}
\widetilde{b}_{1}+\widetilde{b}_{2}= & \left\langle\left[\left(\ddot{a}_{1}+\ddot{a}_{2}, \ddot{b}_{1}+\ddot{b}_{2}, \ddot{c}_{1}+\ddot{c}_{2}\right)\left(\mu_{\ddot{a}_{1}}^{-}+\mu_{\ddot{a}_{2}}^{-}-\mu_{\ddot{a}_{1}}^{-} \mu_{\ddot{a}_{2}}^{-}\right),\right.\right. \\
& \left.\left.\left(\mu_{\ddot{a}_{1}}^{+}+\mu_{\ddot{a}_{2}}^{+}-\mu_{\ddot{a}_{1}}^{+} \mu_{\ddot{a}_{2}}^{+}\right)\right],\left(v_{\ddot{a}_{1}} v_{\ddot{a}_{2}}\right)\right\rangle
\end{aligned}
$$

$$
\begin{aligned}
\tilde{b}_{1}-\widetilde{b}_{2}= & \left\langle\left[\left(\ddot{a}_{1}-\ddot{a}_{2}, \ddot{b}_{1}-\ddot{b}_{2}, \ddot{c}_{1}-\ddot{c}_{2}\right)\left(\mu_{\ddot{a}_{1}}^{-}-\mu_{\ddot{a}_{2}}^{-}+\mu_{\ddot{a}_{1}}^{-} \mu_{\ddot{a}_{2}}^{-}\right),\right.\right. \\
& \left.\left.\left(\mu_{\ddot{a}_{1}}^{+}-\mu_{\ddot{a}_{2}}^{+}+\mu_{\ddot{a}_{1}}^{+} \mu_{\ddot{a}_{2}}^{+}\right)\right],\left(v_{\ddot{a}_{1}} v_{\ddot{a}_{2}}\right)\right\rangle
\end{aligned}
$$

$\left.\left.\left.\lambda \widetilde{b}_{1}=\left\langle\left[\lambda \ddot{a}_{1}, \lambda \ddot{b}_{1}, \lambda \ddot{c}_{1}\right) ; 1-\left(1-\mu_{\ddot{a}_{1}}^{-}\right)^{\lambda}\right), 1-\left(1-\mu_{\ddot{a}_{1}}^{+}\right)^{\lambda}\right], v_{\ddot{a}_{1}}^{\lambda}\right)^{\lambda}\right\rangle$

$\left.\left.\left.\ddot{a}_{1}^{\lambda}=\left\langle\left[\left(\ddot{a}_{1}\right)^{\lambda},\left(\ddot{b}_{1}\right)^{\lambda},\left(\ddot{c}_{1}\right)^{\lambda}\right) ;\left(\mu_{\ddot{a}_{1}}^{-}\right)^{\lambda},\left(\mu_{\ddot{a}_{1}}^{+}\right)^{\lambda}\right], 1-\left(1-v_{\ddot{a}_{1}}\right)^{\lambda}\right)\right)\right\rangle$ 
Example Let

$$
\widetilde{b}_{1}=\langle[((0.4,0.8,0.12) ;[0.24,0.26)], 0.23)\rangle
$$

$$
\widetilde{b}_{2}=\langle[((0.2,0.6,0.10)[0.18,0.20)], 0.18)\rangle
$$

be two triangular cubic fuzzy numbers, then

and

(1):

$$
\begin{aligned}
\widetilde{b}_{1}+\widetilde{b}_{2}= & \langle[(0.4+0.2,0.8+0.6,0.12+0.10)(0.24+0.18-(0.24)(0.18), \\
& (0.26+0.20-(0.26)(0.20)],(0.23)(0.18)\rangle \\
= & {[0.6,1.4,0.22] ;\langle[0.3768,0.408], 0.0414\rangle }
\end{aligned}
$$

(2):

$$
\begin{aligned}
\widetilde{b}_{1}+\widetilde{b}_{2}= & \langle[(0.4-0.2,0.8-0.6,0.12-0.10)(0.24-0.18+(0.24)(0.18), \\
& (0.26-0.20+(0.26)(0.20)],(0.23)(0.18)\rangle \\
= & {[0.2,0.2,0.02] ;\langle[0.1032,0.112], 0.0414\rangle }
\end{aligned}
$$

(3):

$$
\begin{aligned}
\lambda & =0.25,0.25,0.50 \\
\lambda \widetilde{b_{1}} & \left.\left.\left.=\left\langle[0.4 \times 0.25,0.8 \times 0.25,0.12 \times 0.50) ; 1-(1-0.24)^{0.25}\right), 1-(1-0.26)^{0.25}\right], v_{\ddot{u}_{1}}^{\lambda}\right)^{\lambda}\right\rangle \\
& =[0.1,0.2,0.06] ;\langle[0.0663,0.0725], 0.4795\rangle
\end{aligned}
$$

(4):

$$
\begin{aligned}
\widetilde{b}_{1}^{\lambda} & \left.\left.\left.=\left\langle\left[(0.4)^{0.25},(0.8)^{0.25},(0.12)^{0.50}\right) ;(0.24)^{0.25},(0.26)^{0.25}\right], 1-(1-0.23)^{0.50}\right)\right)\right\rangle \\
& =[0.7952,0.9457,0.3464],\langle[0.6999,0.7140], 0.1225\rangle
\end{aligned}
$$

\section{Aggregation Operators on Triangular Cubic Fuzzy Numbers}

Definition Let $\widetilde{b}_{j}(j=1, \ldots, n)$ be a set of triangular cubic fuzzy numbers and TC -WAA : $\Omega_{n} \rightarrow \Omega$; if TC -WAA ${ }_{\omega}\left(\widetilde{b}_{1}, \widetilde{b}_{2}, \ldots, \widetilde{b}_{n}\right)=\sum_{j=1}^{n} w_{j} \widetilde{b}_{j}$ where $\Omega$ is the set of all triangular cubic fuzzy numbers and $\omega=\left(\omega_{1}, \omega_{2}, \ldots, \omega_{n}\right)^{T}$ is the weight vector of $\widetilde{b}_{j}(j=1, \ldots, n), \omega_{j} \in[0,1], \sum_{j=1}^{n} \omega_{j}=1$,

then, TC-WAA is called the weighted arithmetic average operator on cubic triangular fuzzy numbers.

Specially, if $\omega=\left(\frac{1}{n}, \frac{1}{n}, \ldots . \frac{1}{n}\right)^{T}$. TC-WAA is the arithmetic average operator (TC-WA) on triangular cubic fuzzy numbers.
Example Let

$$
\begin{gathered}
{[0.20,0.22,0.23]\langle[0.10,0.12], 0.11\rangle} \\
{[0.1,0.2,0.3]\langle[0.5,0.7], 0.6\rangle} \\
[0.9,0.10,0.11]<[0.45,0.47], 0.46\rangle \\
{[0.30,0.32,0.33]\langle[0.75,0.77], 0.76\rangle} \\
w_{j}=0.25,0.25,0.25,0.25 \\
\left.\widetilde{T C}_{\omega}-\widetilde{b}_{1}, \widetilde{b}_{2}, \ldots, \widetilde{b}_{n}\right)=[0.2710,0.1937,0.2237] \\
;\langle[0.5012,0.5378], 0.3897\rangle \\
\text { Theorem Let } \left.\widetilde{b}=\left\langle\left[\left((\ddot{a}, \ddot{b}, \ddot{c}) ; \mu_{\ddot{a}}^{-}, \mu_{\ddot{a}}^{+}\right)\right], v_{\ddot{a}}\right)\right\rangle \text { be a set of } \\
\text { triangular cubic fuzzy numbers. Then the results aggregated } \\
\text { from definition } 12 \text { above are still triangular cubic fuzzy } \\
\text { numbers and even }
\end{gathered}
$$

$$
\begin{aligned}
\mathrm{TC}-\mathrm{WAA}_{\omega}\left(\widetilde{b}_{1}, \widetilde{b}_{2}, \ldots, \widetilde{b}_{n}\right)= & \sum_{j=1}^{n} w_{j} \ddot{a}_{j}=\left\langle\left[\left(\left(\prod_{j=1}^{n}(\ddot{a})^{\omega_{j}}, \prod_{j=1}^{n}(\ddot{b})^{\omega_{j}}, \prod_{j=1}^{n}(\ddot{c})^{\omega_{j}}\right)\right.\right.\right. \\
& \left.\left.; 1-\prod_{j=1}^{n}\left(1-\mu_{\ddot{a}}^{-}\right)^{\omega_{j}}, 1-\prod_{j=1}^{n}\left(1-\mu_{\ddot{a}}^{+}\right)^{\omega_{j}}\right], ; \prod_{j=1}^{n}\left(v_{\ddot{a}}\right)^{\omega_{j}}\right\rangle
\end{aligned}
$$

where $\omega=\left(\omega_{1}, \omega_{2}, \ldots, \omega_{n}\right)^{T} \quad$ is $\quad$ the $\quad$ weight $\quad$ vector $\quad$ of $\quad \ddot{a}_{j}(j=1, \ldots, n), \omega_{j} \in[0,1], \sum_{j=1}^{n} \omega_{j}=1$. 
Definition Give $\tilde{b}_{j}(j=1, \ldots, n)$ a chance to be an arrangement of cubic triangular fuzzy numbers and TC WGA $: \Omega_{n} \rightarrow \Omega$; if TC -WGA $\omega_{\omega}\left(\widetilde{b}_{1}, \widetilde{b}_{2}, \ldots, \tilde{b}_{n}\right)=\sum_{j=1}^{n} \widetilde{b}_{j}^{w_{j}}$ where $\Omega$ is the set of all triangular cubic fuzzy numbers and $\omega=\left(\omega_{1}, \omega_{2}, \ldots \omega_{n}\right)^{T}$ is the weight vector of $\ddot{a}_{j}(j=1, \ldots, n), \omega_{j} \in[0,1]$ and $\sum_{j=1}^{n} \omega_{j}=1$.

At that point, TC-WAA is known as the weighted arithmetic average operator on triangular cubic fuzzy numbers.

If $\omega=\left(\frac{1}{n}, \frac{1}{n}, \ldots \cdot \frac{1}{n}\right)^{T}$. TC-WAA is the arithmetic average operator (TC-WA) on triangular cubic fuzzy numbers.

Example Let
$[0.17,0.18,0.19] ;\langle[0.7,0.9], 0.8\rangle$

$[0.4,0.5,0.6] ;\langle[0.12,0.14], 0.13\rangle$

$[0.10,0.12,0.14] ;\langle[0.29,0.31], 0.30\rangle$

$[0.22,0.24,0.26] ;\langle[0.50,0.52], 0.51\rangle$

$$
\begin{aligned}
\omega_{j} & =0.25,0.25,0.25,0.25 \\
\text { TC }-\mathrm{WGA}_{\omega}\left(\tilde{b}_{1}, \tilde{b}_{2}, \ldots, \tilde{b}_{n}\right) & =[0.1966,0.2256,0.2538]
\end{aligned}
$$

$;\langle[0.3322,0.3775], 0.5057\rangle$

Theorem Let $\left.\quad \ddot{a}=\left\langle\left[(\ddot{a}, \ddot{b}, \ddot{c}, d) ;\left[\mu_{\ddot{a}}^{-}, \mu_{\ddot{a}}^{+}\right)\right], v_{\ddot{a}}\right)\right\rangle \quad$ be $\quad$ an arrangement of triangular cubic fuzzy numbers. Then the outcomes aggregated from definition 13 are still triangular cubic fuzzy numbers and even

TC-WGA

$$
\left(\tilde{b}_{1}, \tilde{b}_{2}, \ldots \tilde{b}_{n}\right)=\sum_{j=1}^{n} w_{j} \ddot{a}_{j}=\left\{\begin{array}{c}
{\left[\left(\prod_{j=1}^{n}(\ddot{a})^{\omega_{j}}, \prod_{j=1}^{n}(\ddot{b})^{\omega_{j}}, \prod_{j=1}^{n}(\ddot{c})^{\omega_{j}}\right) ; \prod_{j=1}^{n}\left(\mu_{\ddot{a}}^{-}\right)^{\omega_{j}},\right.} \\
\left.\prod_{j=1}^{n}\left(\mu_{\ddot{a}}^{+}\right)^{\omega_{j}}\right] ; \prod_{j=1}^{n}\left(v_{\ddot{a}}\right)^{\omega_{j}}
\end{array}\right\rangle
$$

Where $\omega=\left(\omega_{1}, \omega_{2}, \ldots, \omega_{n}\right)^{T}$ is the weight vector of $\widetilde{b}_{j}(j=1, \ldots, n)$ and $\omega_{j} \in[0,1], \sum_{j=1}^{n} w_{j}=1$

\section{Expected Values of Triangular Cubic Fuzzy Numbers and Comparison Between Them}

For a triangular cubic fuzzy numbers, $f_{L}(x)$ are entirely direct expanding capacity and $f_{R}(x)$ is entirely straight diminishing capacity in definition 1 . Their inverse functions are separately,

$$
p_{\tilde{b}}^{L}(x)=\ddot{a}+\frac{y}{\left[\mu_{\tilde{b}}^{-}, \mu_{\tilde{b}}^{+}\right]} \times(\ddot{b}-\ddot{a}), p_{\widetilde{b}}^{R}(\ddot{b})=\ddot{b}+\frac{y}{\left[\mu_{\tilde{b}}, \mu_{\tilde{b}}^{+}\right]} \times(\ddot{c}-\ddot{b}),
$$

The trust degree of triangular cubic fuzzy number $\widetilde{b}$ is between $\left.\left\langle\left[\mu_{\widetilde{b}}^{-}, \mu_{\widetilde{b}}^{+}\right], 1-v_{\widetilde{b}}\right)\right\rangle$.

Definition

$$
\left.\left.\left.\left.I_{\lambda}(\tilde{b})=\frac{1}{3} \int_{0}^{\left[\mu_{\tilde{b}}^{\bar{\sigma}}, \mu_{\tilde{b}}^{+}\right]}(1-\lambda) \times g_{\widetilde{b}}^{L}(x)+\lambda \times g_{\widetilde{b}}^{R}(x)\right]\right\} d y+\int_{0}^{1-v_{\tilde{b}}}(1-\lambda) \times g_{\widetilde{b}}^{L}(x)+\lambda \times g_{\widetilde{b}}^{R}(x)\right]\right\} d y
$$

is known as the expected value of triangular cubic fuzzy number $\tilde{b}$.

Theorem For the triangular cubic fuzzy number

$$
\left.\widetilde{a}=\left\langle\left[\left((\ddot{a}, \ddot{b}, \ddot{c}) ; \mu_{\ddot{a}}^{-}, ; \mu_{\ddot{a}}^{+}\right)\right], v_{\ddot{a}}\right)\right\rangle, I(\widetilde{b})=\frac{1}{9}\left\langle\left[\left((\ddot{a}+\ddot{b}+\ddot{c}) \times\left[1+\mu_{\widetilde{b}}^{-}-v_{\widetilde{b}}\right) \times\left(1+\mu_{\widetilde{b}}^{+}-v_{\widetilde{b}}\right)\right\rangle .\right.\right.
$$

Example Give $\widetilde{b}=\langle[((0.5,0.9,0.13) ;[0.28,0.30)], 0.29)\rangle$ be the triangular cubic fuzzy numbers, then

$$
\begin{aligned}
& I(\tilde{b})=\frac{1}{9}\langle[((0.5+0.9+0.13) \times[1+0.28-0.29) \times(1+0.30-0.29)\rangle \\
& =\frac{1}{9}\left\langle[(1.53) \times[0.99) \times(1.01)\rangle=\frac{1.529847}{9}=0.1699 .\right.
\end{aligned}
$$

Definition Give $\left.\tilde{b}=\left\langle\left[\left((\ddot{a}, \ddot{b}, \ddot{c}) ; \mu_{\tilde{b}}^{-}, \mu_{\tilde{b}}^{+}\right)\right], v_{\tilde{b}}\right)\right\rangle$ a chance to be a 
Example let $\tilde{b}=\langle[((0.9,0.11,0.13) ;[0.4,0.6], 0.5\rangle$ a chance to be a triangular cubic fuzzy number. Then $S(\tilde{b})=I_{\tilde{b}} \times\left[\left(\mu_{\tilde{b}}^{-}, \mu_{\tilde{b}}^{+}\right)-\left(1-v_{\tilde{b}}\right)\right]$ is known the score function of $\widetilde{a}$, where $I_{\ddot{a}}$ is the expected value of triangular cubic fuzzy number $\ddot{a}$.

$$
\begin{aligned}
I_{\widetilde{b}} & =0.254 \\
S(\widetilde{b}) & =0.254 \times[0.5] \\
S(\widetilde{b}) & =0.127
\end{aligned}
$$

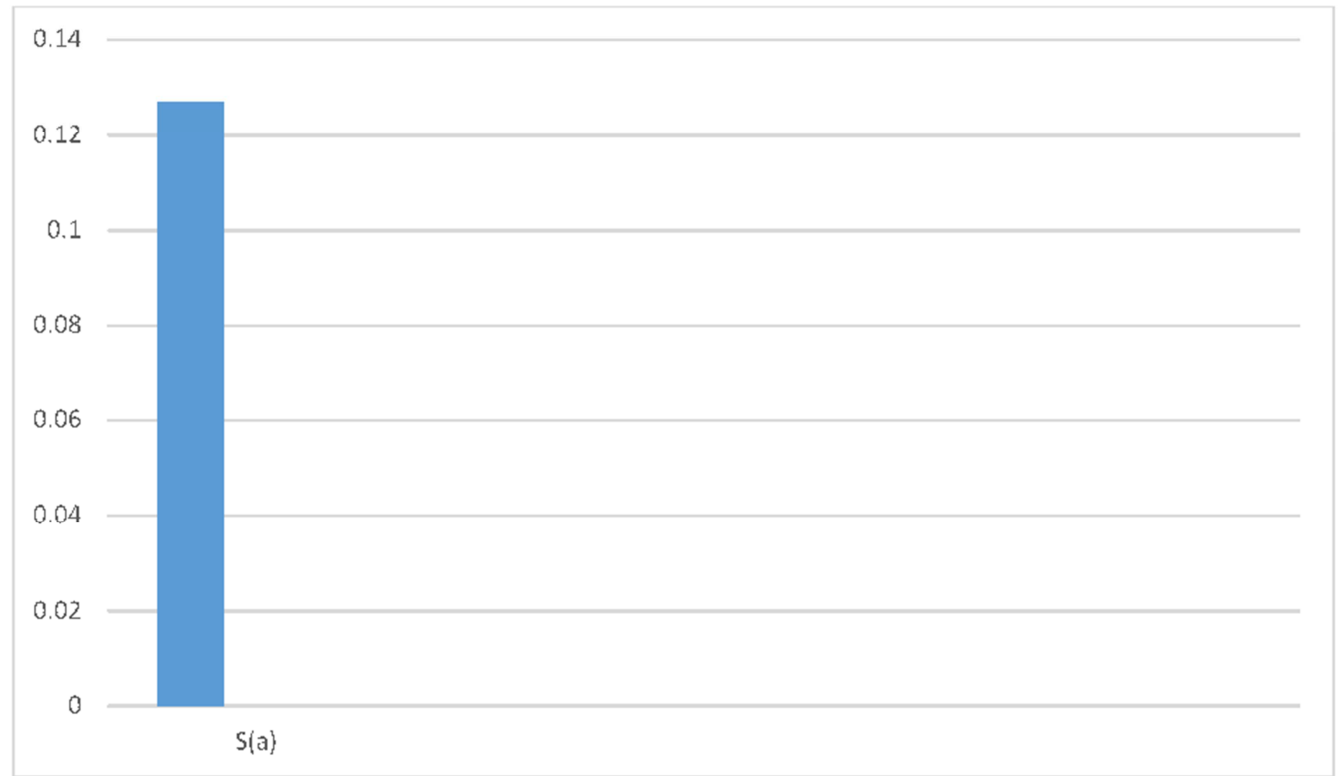

Figure 1. Different value of alternatives.

Definition Give $\left.\tilde{b}=\left\langle\left[(\ddot{a}, \ddot{b}, \ddot{c}) ;\left[\mu_{\tilde{b}}^{\bar{b}}, \mu_{\tilde{b}}^{+}\right)\right], v_{\tilde{b}}\right)\right\rangle$ a chance to be a triangular cubic fuzzy number. Then $H(\widetilde{b})=I_{\tilde{b}} \times\left[\left(\mu_{\tilde{b}}^{-}, \mu_{\tilde{b}}^{+}\right)+\left(1-v_{\tilde{b}}\right)\right]$ is known the accuracy function of $\tilde{b}$, where $I_{\widetilde{b}}$ is the expected value of triangular cubic fuzzy number $\tilde{b}$.

Example Let $\tilde{b}=\langle[((0.1,0.2,0.3) ;[0.15,0.17], 0.16\rangle$ a chance to be a triangular cubic fuzzy number. Then $S(\tilde{b})=I_{\tilde{b}} \times\left[\left(\mu_{\tilde{b}}^{-}, \mu_{\tilde{b}}^{+}\right)+\left(1-v_{\tilde{b}}\right)\right]$ is known the score function of $\widetilde{b}$, where $I_{\widetilde{b}}$ is the expected value of triangular cubic fuzzy number $\tilde{b}$.

$$
\begin{aligned}
I_{\widetilde{b}} & =0.6 \times 0.99 \times 1.01 \\
I_{\widetilde{b}} & =0.0666 \\
S(\tilde{b}) & =0.0666 \times[0.32)+0.84] \\
S(\tilde{b}) & =0.0772
\end{aligned}
$$

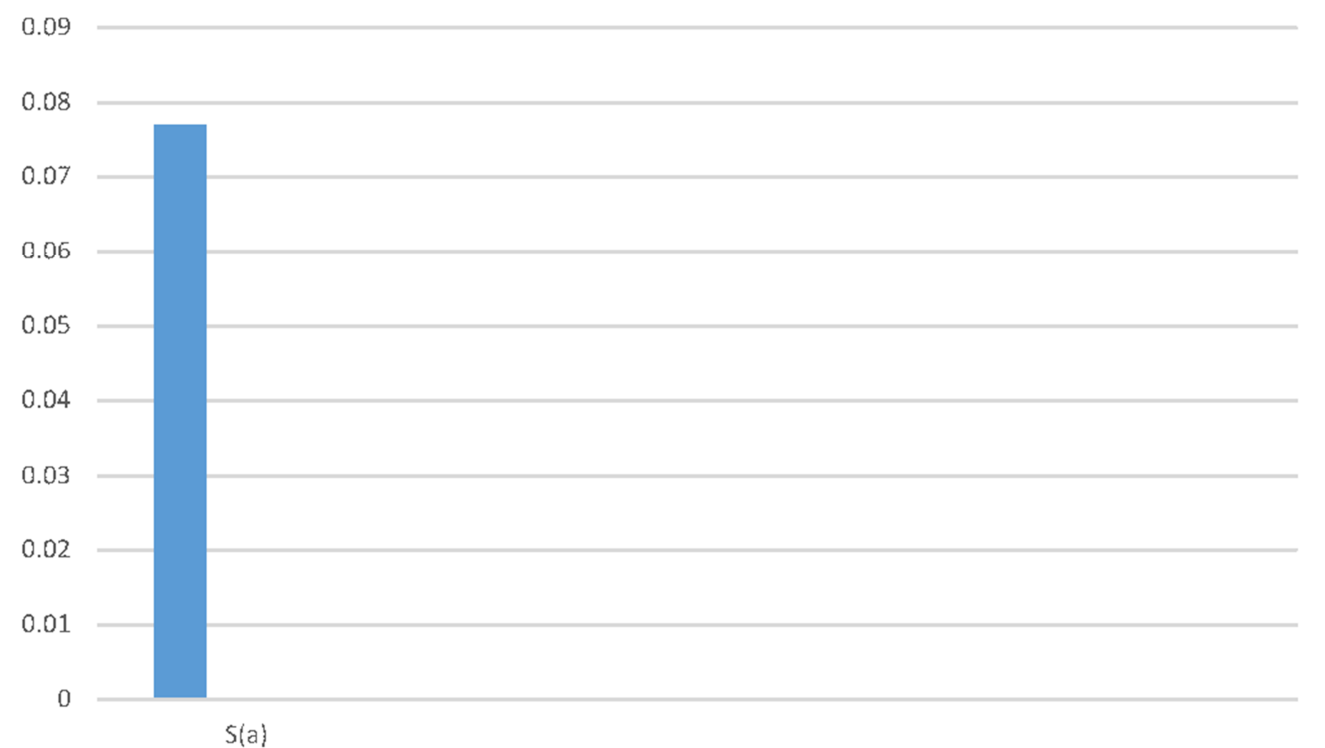

Figure 2. Ranking of score value. 


\section{Multi-Criteria Decision Making Method Based on Triangular Cubic Fuzzy Numbers}

For some fuzzy multi-criteria decision making problem, assume that there are $\mathrm{m}$ alternatives $\ddot{a}=\left\{\ddot{a}_{1}, \ddot{a}_{2}, \ldots, \ddot{a}_{n}\right\}, l$ decision criteria $\ddot{c}=\left\{\ddot{c}_{1}, \ddot{c}_{2}, \ldots, \ddot{c}_{n}\right\}$ and the corresponding weight coefficients are $\omega=\left\{\omega_{1}, \omega_{2}, \ldots, \omega_{l}\right\}, \omega_{j} \in[0,1], \omega_{1}+\omega_{2}+\ldots+\omega_{l}=1$. The value of alternative ai on the criteria $\ddot{c}_{j}$ is cubic triangular cubic fuzzy number $\ddot{a}_{i j}=\left\langle\left(\left[m_{1 j}\left(\ddot{a}_{i}\right), m_{2 j}\left(\ddot{a}_{i}\right), m_{3 j}\left(\ddot{a}_{i}\right)\right] ;\left[\mu_{j}^{-}\left(\ddot{a}_{i}\right), \mu_{j}^{+}\left(\ddot{a}_{i}\right)\right], v_{j}\left(\ddot{a}_{i}\right)\right\rangle\right.$.

The familiar types of criteria are benefit and cost in multi criteria decision making problems. To eliminate the effect from different physical dimensions to decision results, the matrix $T=\left(t_{i j}\right)_{m \times n}$,

$$
\left(t_{i j}\right)_{m \times n}=\left\langle\left(\left[m_{1 j}\left(\ddot{a}_{i}\right), m_{2 j}\left(\ddot{a}_{i}\right), m_{3 j}\left(\ddot{a}_{i}\right)\right] ;\left[\mu_{j}^{-}\left(\ddot{a}_{i}\right), \mu_{j}^{+}\left(\ddot{a}_{i}\right)\right], v_{j}\left(\ddot{a}_{i}\right)\right\rangle\right.
$$

Composed by triangular cubic fuzzy numbers of fuzzy decision matrix $D=\left(\ddot{a}_{i j}\right)_{n \times l}$ is translated into assimilate matrix $R=\left(r_{i j}\right)_{n \times l}, r_{i j}=\left\langle\left[r_{i j}^{1}, r_{i j}^{2}, r_{i j}^{3}\right]\right\rangle$ using formulas to assimilate the triangular cubic fuzzy decision matrix.

For cost criteria:

$$
r_{i j}^{k}=\frac{\max _{j}\left(m_{4 j}\left(\ddot{a}_{i}\right)\right)-m_{k j}\left(\ddot{a}_{i}\right)}{\max _{j}\left(m_{4 j}\left(\ddot{a}_{i}\right)\right)-\min _{j}\left(m_{1 j}\left(\ddot{a}_{i}\right)\right)}, k=1, \ldots ., 4
$$

For benefit criteria:

$$
r_{i j}^{k}=\frac{m_{k j}\left(\ddot{a}_{i}\right)-\min _{j}\left(m_{4 j}\left(\ddot{a}_{i}\right)\right)}{\max _{j}\left(m_{4 j}\left(\ddot{a}_{i}\right)\right)-\min _{j}\left(m_{1 j}\left(\ddot{a}_{i}\right)\right)}, k=1, \ldots, 4
$$

Decision steps:

(1) Assimilate decision matrix

(2) Using weighted arithmetic average operator

$\ddot{a}_{i}=$ TC-WAA ${ }_{\omega}\left(\ddot{c}_{1}\left(\ddot{a}_{i}\right), \ddot{c}_{2}\left(\ddot{a}_{i}\right), \ldots, \ddot{c}_{l}\left(\ddot{a}_{i}\right)\right), i=1,2, \ldots, n$

or using weighted geometric average operator $\ddot{a}_{i}=\mathrm{TC}-\mathrm{WGA}{ }_{\omega}\left(\ddot{c}_{1}\left(\ddot{a}_{i}\right), \ddot{c}_{2}\left(\ddot{a}_{i}\right), \ldots, \ddot{c}_{l}\left(\ddot{a}_{i}\right)\right), i=1,2, \ldots, n$

Total criteria's weights and values to accomplish the incorporated triangular cubic fuzzy numbers $\ddot{a}_{i}, i=1,2, \ldots, n$ of alternative $\ddot{a}_{i}$.

(3) Compute the score esteem and the accuracy esteem utilizing the score work and the accuracy work, separately.

(4) Rank the alternatives by definition 15.

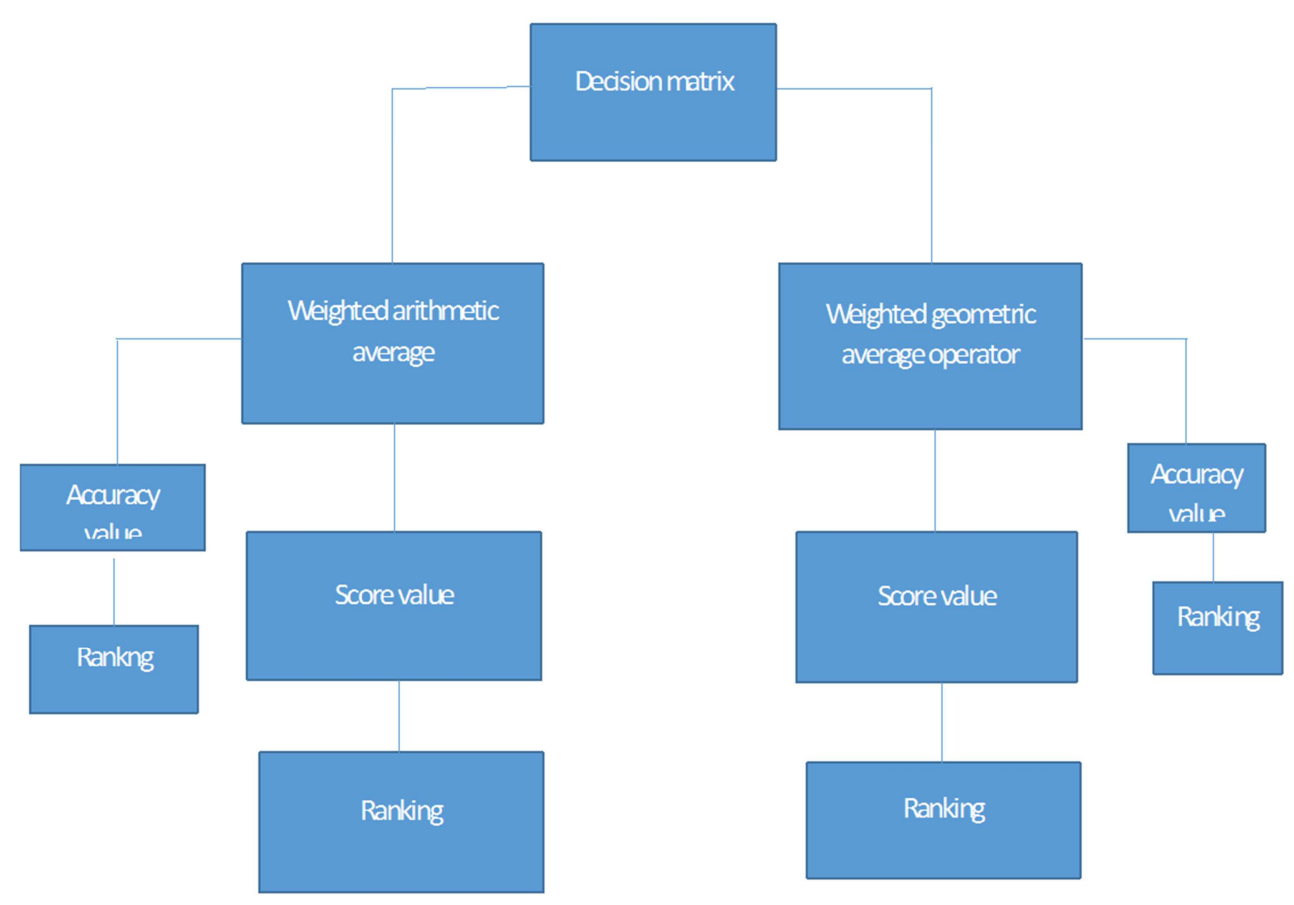

Figure 3. Proposed method

\section{Numerical Application}

There are 4 alternatives $\ddot{a}_{1}, \ddot{a}_{2}, \ldots, \ddot{a}_{4}$ and 4 criteria $\ddot{c}_{1}, \ddot{c}_{2}, \ldots, \ddot{c}_{4}$ in a multi-criteria decision making problem; the weight vector of criteria is $\omega=(0.2,0.3,0.4,0.1)$, and the decision information is given by decision makers, trying to get ranking of the 4 alternatives.

Steps utilizing the strategy in this article are as per the following

(1) Standardize data in decision matrix;

Decision matrix 1 


$$
\begin{gathered}
c_{1} \\
A_{1}\left\{\begin{array}{l}
c_{2} \\
\{0.3,0.5, \\
0.6] ;\langle[0.2, \\
0.8], 0.5\rangle
\end{array}\right\}\left\{\begin{array}{l}
{[0.14,0.18,} \\
0.20] ;\langle[0.11, \\
0.20], 0.16\rangle
\end{array}\right\}\left\{\begin{array}{l}
c_{3} \\
0.1,0.4, \\
0.5] ;\langle[0.2, \\
0.10], 0.7\rangle
\end{array}\right\}\left\{\begin{array}{l}
c_{4} \\
0.42,0.44, \\
0.46] ;\langle[0.32, \\
0.40], 0.35\rangle
\end{array}\right\} \\
A_{2}\left\{\begin{array}{l}
{[0.1,0.3,} \\
0.8] ;\langle[0.4, \\
0.10], 0.7\rangle
\end{array}\right\}\left\{\begin{array}{l}
{[0.2,0.4,} \\
0.6] ;\langle[0.3, \\
0.9], 0.5\rangle
\end{array}\right\}\left\{\begin{array}{l}
{[0.12,0.14,} \\
0.18] ;\langle[0.2, \\
0.7], 0.5\rangle
\end{array}\right\}\left\{\begin{array}{l}
{[0.19,0.24,} \\
0.29] ;\langle[0.12, \\
40], 0.30\rangle
\end{array}\right\} \\
A_{3}\left\{\begin{array}{l}
{[0.2,0.4,} \\
0.6] ;\langle[0.12, \\
0.16], 0.14\rangle
\end{array}\right\}\left\{\begin{array}{l}
{[0.4,0.7,} \\
0.9] ;\langle[0.8, \\
0.12], 0.10\rangle
\end{array}\right\}\left\{\begin{array}{l}
{[0.22,0.32,} \\
0.34] ;\langle[0.11, \\
0.19], 0.16\rangle
\end{array}\right\}\left\{\begin{array}{l}
{[0.4,0.8,} \\
0.6] ;\langle[0.42, \\
0.60], 0.56\rangle
\end{array}\right\} \\
A_{4}\left\{\begin{array}{l}
{[0.5,0.7,} \\
0.9] ;\langle[0.2, \\
0.8], 0.5\rangle
\end{array}\right\}\left\{\begin{array}{l}
{[0.4,0.6,} \\
0.10] ;\langle[0.11, \\
0.20], 0.16\rangle
\end{array}\right\}\left\{\begin{array}{l}
{[0.11,0.15,} \\
0.16] ;\langle[0.2, \\
0.10], 0.7\rangle
\end{array}\right\}\left\{\begin{array}{l}
{[0.12,0.14,} \\
0.16] ;\langle[0.32, \\
0.40], 0.35\rangle
\end{array}\right\}
\end{gathered}
$$

(2) Aggregate all the elements aij $(j=1, \ldots, 4)$ in the ith row of decision matrix $D$ using TC-WAA; then, the combined triangular cubic fuzzy numbers $\ddot{a}_{i}, i=1,2, \ldots, 4$ of alternative $\ddot{a}_{i}$ are attained TC-WAA

$$
\begin{array}{ll}
A_{1} & \{[0.192,0.304,0.354],\langle[0.1727,0.3872], 0.4554\rangle\} \\
A_{2} & \{[0.183,0.324,0.561],\langle[0.3061,0.7096], 0.4130\rangle\} \\
A_{3} & \{[0.488,0.888,0.976],\langle[0.6168,0.4354], 0.0888\rangle\} \\
A_{4} & \{[0.113,0.159,0.132],\langle[0.1727,0.3872], 0.4554\rangle\}
\end{array}
$$

(3) Enumerate the score values $S\left(\ddot{a}_{i}\right)$ of $\ddot{a}_{i}$

$$
S_{1}=0.0065, S_{2}=0.0828, S_{3}=0.5180, S_{4}=0.0031
$$

(4) Rank the alternatives by

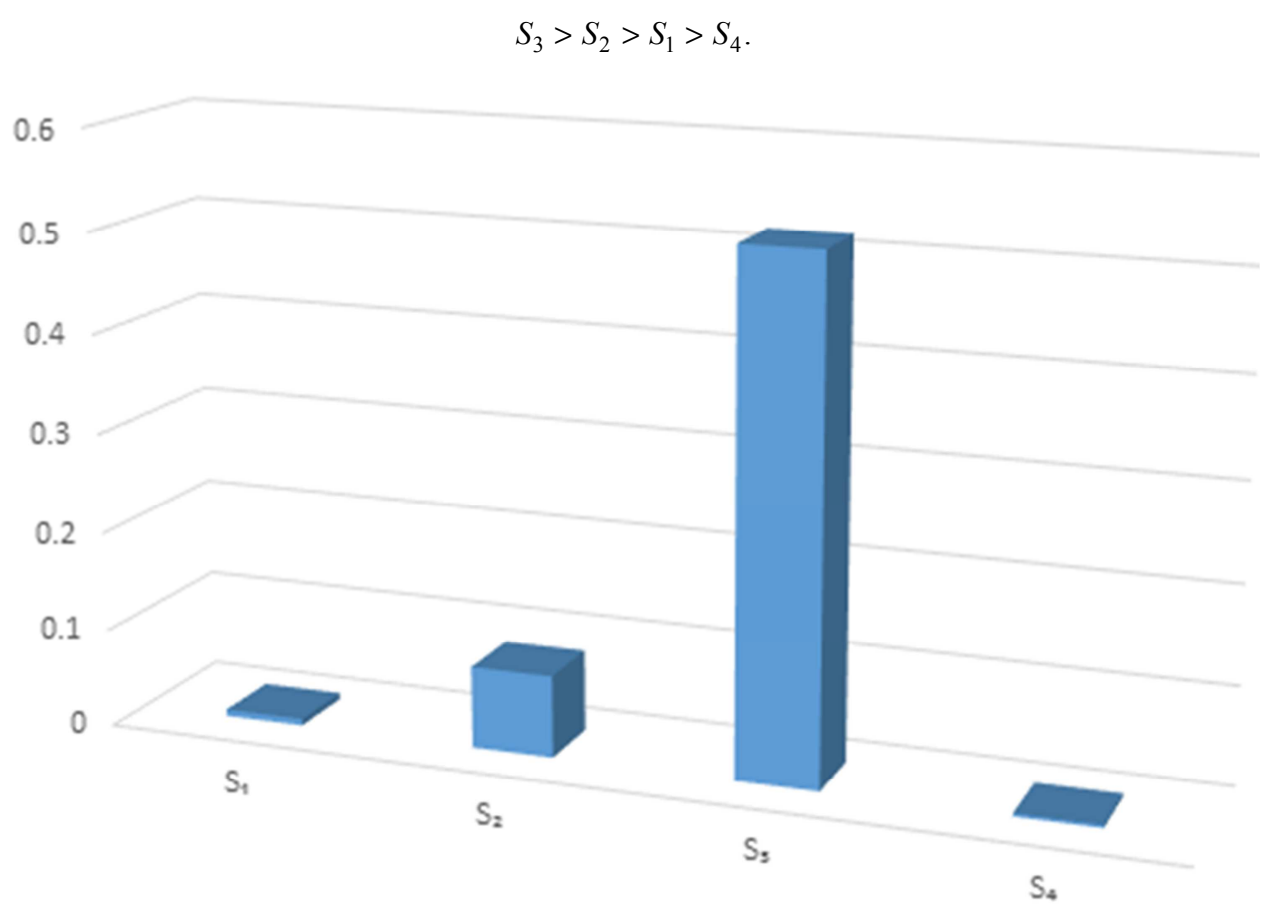

Figure 4. Raking of alternatives 
Enumerate the accuracy values $H\left(\ddot{a}_{i}\right)$ of $\ddot{a}_{i}$

$$
H_{1}=0.0696, H_{2}=0.2202, H_{3}=1.0557, H_{4}=0.0331
$$

\section{Accuracy value}

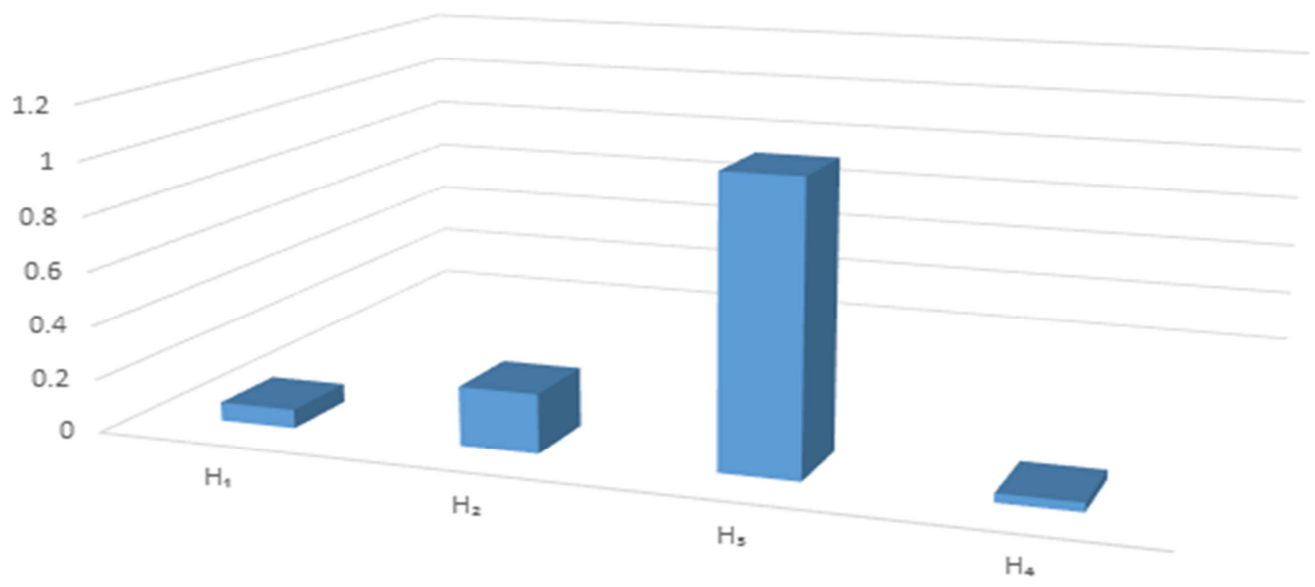

Figure 5. Accuracy value of different ranking

Decision matrix

$$
\begin{gathered}
c_{1} \\
A_{1}\left\{\begin{array}{l}
{[0.3,0.5,} \\
0.6] ;\langle[0.2,\} \\
0.8], 0.5\rangle
\end{array}\right\}\left\{\begin{array}{l}
c_{2}[0.14,0.18, \\
0.20] ;\langle[0.11, \\
0.20], 0.16\rangle
\end{array}\right\}\left\{\begin{array}{l}
{[0.1,0.4,} \\
0.5] ;\langle[0.2,\} \\
0.10], 0.7\rangle
\end{array}\right\}\left\{\begin{array}{l}
c_{4} \\
0.46] ;\langle[0.44, \\
0.40], 0.35\rangle
\end{array}\right\} \\
A_{2}\left\{\begin{array}{l}
{[0.1,0.3,} \\
0.8] ;\langle[0.4, \\
0.10], 0.5\rangle
\end{array}\right\}\left\{\begin{array}{l}
{[0.2,0.4,} \\
0.6] ;\langle[0.3, \\
0.9], 0.4\rangle
\end{array}\right\}\left\{\begin{array}{l}
{[0.12,0.14,} \\
0.18] ;\langle[0.2, \\
0.7], 0.3\rangle
\end{array}\right\}\left\{\begin{array}{l}
{[0.19,0.24,} \\
0.29] ;\langle[0.12, \\
40], 0.15\rangle
\end{array}\right\} \\
A_{3}\left\{\begin{array}{l}
{[0.2,0.4,} \\
0.6] ;\langle[0.4, \\
0.10], 0.15\rangle
\end{array}\right\}\left\{\begin{array}{l}
{[0.4,0.7,} \\
0.9] ;\langle[0.3, \\
0.9], 0.4\rangle
\end{array}\right\}\left\{\begin{array}{l}
{[0.22,0.32,} \\
0.34] ;\langle[0.2, \\
0.7], 0.3\rangle
\end{array}\right\}\left\{\begin{array}{l}
{[0.4,0.8,} \\
0.6] ;\langle[0.12, \\
0.40], 0.15\rangle
\end{array}\right\} \\
A_{4}\left\{\begin{array}{l}
{[0.5,0.7,} \\
0.9] ;\langle[0.2, \\
0.8], 0.5\rangle
\end{array}\right\}\left\{\begin{array}{l}
{[0.4,0.6,} \\
0.10] ;\langle[0.11, \\
0.20], 0.16\rangle
\end{array}\right\}\left\{\begin{array}{l}
{[0.11,0.15,} \\
0.16] ;\langle[0.2, \\
0.10], 0.7\rangle
\end{array}\right\}\left\{\begin{array}{l}
{[0.12,0.14,} \\
0.16] ;\langle[0.32, \\
0.40], 0.35\rangle
\end{array}\right\}
\end{gathered}
$$

If all elements $\ddot{a}_{i j}(j=1, \ldots, 4)$ in the ith row of decision matrix $D$ are aggregated using TC-WGA, the integrated $A_{1} \quad\{[0.281,0.394,0.487],\langle[0.2689,0.3642], 0.3937\rangle\}$ $A_{2}\{[0.099,0.192,0.330],\langle[0.1729,0.3315], 0.4036\rangle\}$ $A_{3}\{[0.137,0.310,0.413],\langle[0.1729,0.3315], 0.4036\rangle\}$ $A_{4}\{[0.552,0.623,0.544],\langle[0.5187,0.6034], 0.2213\rangle\}$

(3) Aggregate all the elements a $i j(j=1, \ldots, 4)$ in the ith row of decision matrix $D$ using TC-WGA; then, the concentrate triangular cubic fuzzy numbers $\ddot{a}_{i}, i=1,2, \ldots, 4$ of alternative $\ddot{a}_{i}$ are attained.

TC-WGA

(3) Calculate the score values $S\left(\ddot{a}_{i}\right)$ of $\ddot{a}_{i}$

$$
S_{1}=0.0262, S_{2}=0.0049, S_{3}=0.0069, S_{4}=0.2905
$$

(4) Rank the alternatives by

$$
S_{4}>S_{1}>S_{3}>S_{2}
$$




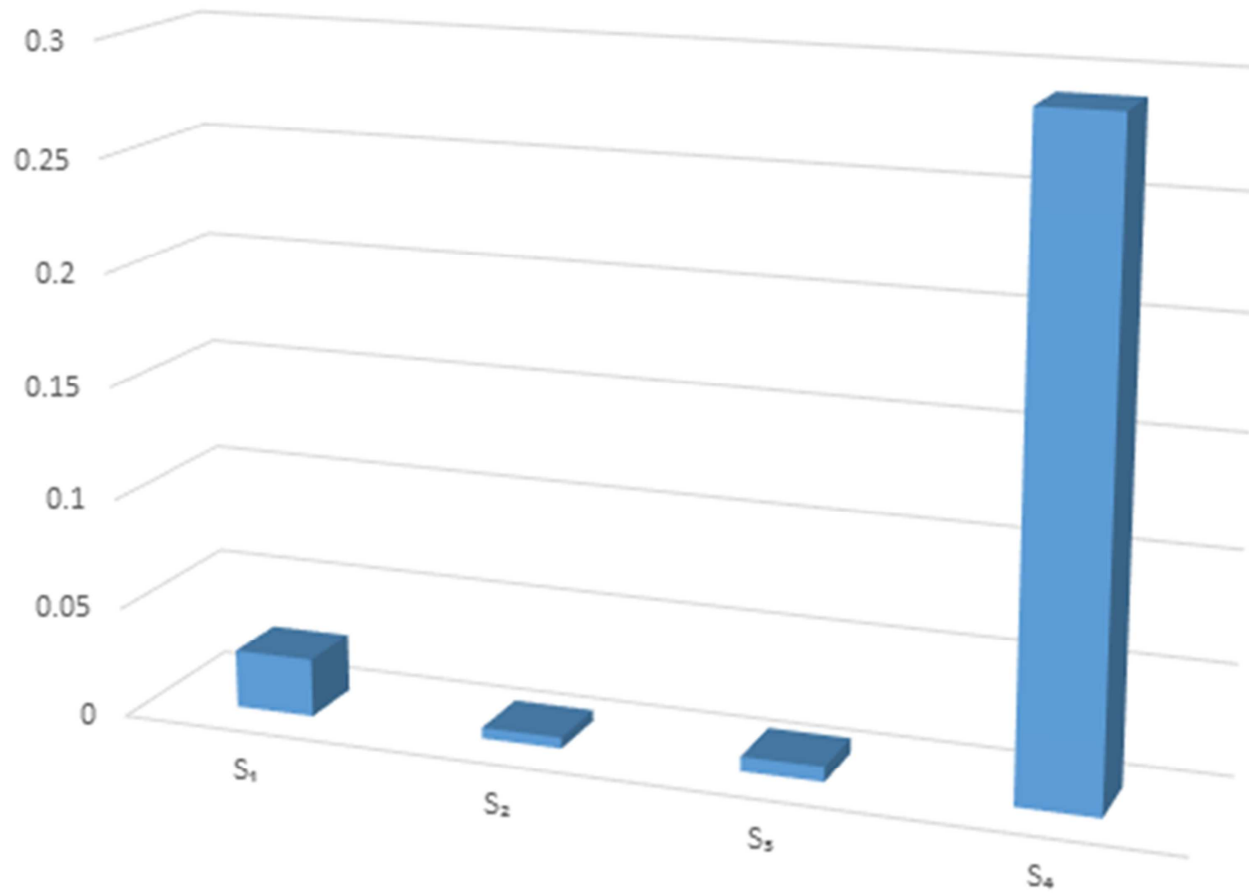

Figure 6. Different value of aggregate value

Enumerate the accuracy values $H\left(\ddot{a}_{i}\right)$ of $\ddot{a}_{i}$

$$
H_{1}=0.1358, H_{2}=0.0541, H_{3}=0.0750, H_{4}=0.6508 \text {. }
$$

\section{Accuracy value}

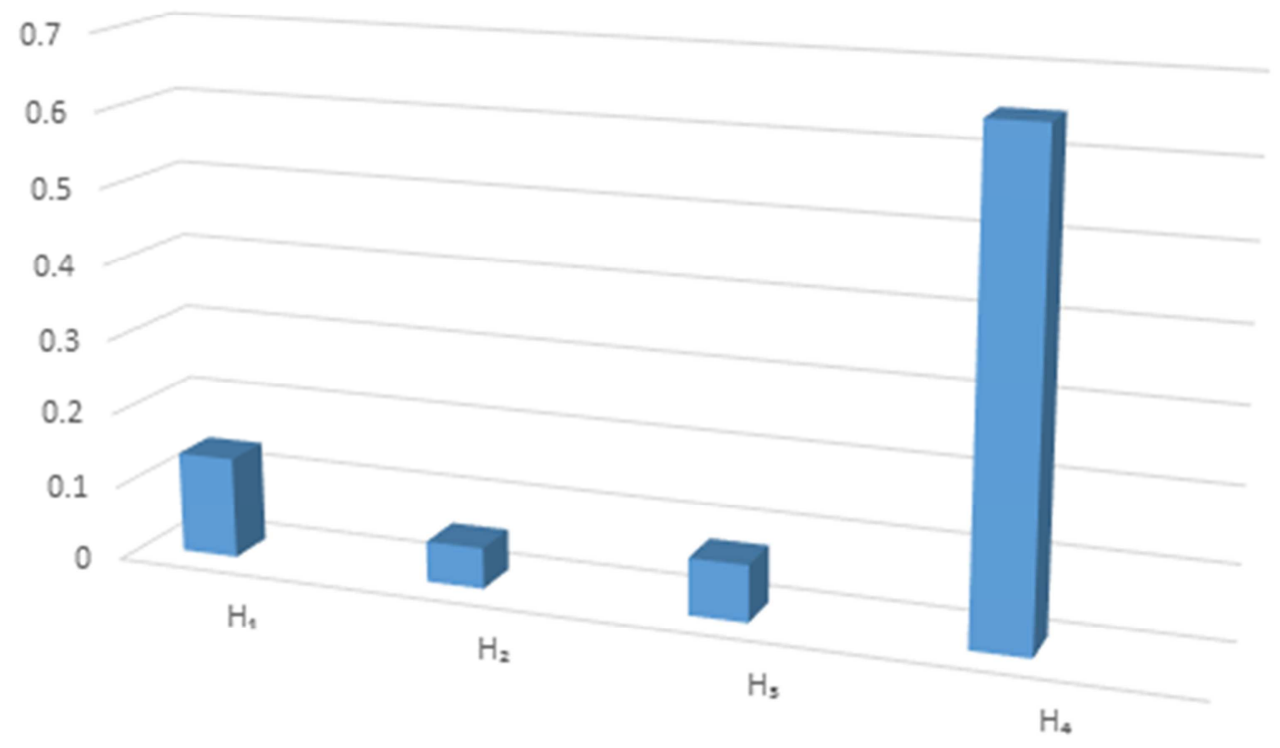

Figure 7. Different value of accuracy values

\section{Conclusion}

Aggregation strategies on triangular cubic fuzzy numbers are deliberated in this article, based at the definitions of cubic fuzzy numbers and triangular cubic fuzzy numbers. A technique to equate triangular cubic fuzzy numbers is given and carried out to selection making. The imaginative method to solve fuzzy multi-criteria decision making problems based on cubic triangular fuzzy numbers is furnished. 


\section{References}

[1] F. Amin, A. Fahmi, S. Abdullah,A. Ali, R. Ahmed and F. Ghanu. Triangular cubic linguistic hesitant fuzzy aggregation operators and their application in group decision making, Journal of Intelligent and Fuzzy System, 34, (2018) 2401-2416.

[2] A. Fahmi, S. Abdullah, F. Amin, N. Siddiqui and A. Ali. Aggregation operators on triangular cubic fuzzy numbers and its application to multi-criteria decision making problems. Journal of Intelligent \& Fuzzy Systems, 33,(2017), 33233337.

[3] A. Fahmi, S. Abdullah, F. Amin \& A. Ali (2017). Precursor Selection for Sol--Gel Synthesis of Titanium Carbide Nanopowders by a New Cubic Fuzzy Multi-Attribute Group Decision-Making Model. Journal of Intelligent Systems (2017).

[4] A. Fahmi, S. Abdullah, F. Amin \& A. Ali, A. Weighted Average Rating (War) Method for Solving Group Decision Making Problem Using Triangular Cubic Fuzzy Hybrid Aggregation (Tcfha). Punjab University Journal of Mathematics, 50(1), (2018), 23-34.

[5] A. Fahmi, S. Abdullah, F. Amin, A. Ali and W.A. Khan. Some geometric operators with Triangular Cubic Linguistic Hesitant Fuzzy number and Their Application in Group DecisionMaking, Journal of Intelligent and Fuzzy System, accept (2018).

[6] A. Fahmi, S. Abdullah and F. Amin. TRAPEZOIDAL LINGUISTIC CUBIC HESITANT FUZZY TOPSIS METHOD AND APPLICATION TO GROUP DECISION MAKING PROGRAM. 19, (2017), 27-47.

[7] A. Fahmi, S. Abdullah and F. Amin. Expected Values of Aggregation Operators on Cubic Trapezoidal Fuzzy Number and its Application to Multi-Criteria Decision Making Problems. 22, (2018) 51-65.

[8] A. Fahmi, S. Abdullah, F. Amin and M. S. A. Khan. Trapezoidal cubic fuzzy number Einstein hybrid weighted averaging operators and its application to decision making, soft computing, (2018) DOI: 10.1007/s00500-018-3242-6.

[9] T. Aouam, Chang S I, Lee E S. Fuzzy MADM: an outranking method. European Journal of Operational Research, 2003, 145: 317-328.

[10] K. T. Atanassov, Intuitionistic Fuzzy Sets, Fuzzy Sets Syst., 20 (1), 87--96:(1986).

[11] T. Chu, A fuzzy number interval arithmetic based fuzzy MCDM algorithm. Inter. J. Fuzzy Systems, 2002, 4(4): 867872.

[12] S. K. De, Biswas R, Roy A R. An application of intuitionistic fuzzy sets in medical diagnosis. Fuzzy Sets and Systems, 2001, 117(2): 209-213.

[13] Y. B. Jun, C. S. Kim and Ki. O. Yang, Cubic sets "Annals of Fuzzy Mathematics and Informatics,", Volume 4, No. 1, 8398: (2012).

[14] R J. Li, Theories and applications of fuzzy multi-criteria decision making. Beijing: Science Press, 2002.

[15] D F. Li. Multiattribute decision making models and methods using intuitionistic fuzzy sets. J.Comput.System Sci., 2005, 70: 73-85.

[16] G.Przemysiaw Distances between intuitionistic fuzzy sets and/or interval-valued fuzzy sets based on the Hausdorff metric. Fuzzy Sets and Systems, 2004, 148(2):319-328.

[17] M. H. Shu, Cheng C H, Chang J R. Using intuitionistic fuzzy sets for fault-tree analysis on printed circuit board assembly. Microelectronics Reliability, 2006(46): 2139-2148.

[18] J, Q. Wang, Programming method of fuzzy group multiple criteria decision making with incomplete information. Systems Engineering and Electronics, 2004, 26(11): 16041608.

[19] J Q. Wang, Study on multi-criteria decision-making approach with incomplete certain information. Central South University, 2005.

[20] J Wang, Zhang Z. Compromise approach on multicriteria intuitionistic fuzzy decision-making with incomplete certain information. Chinese Control and Decision Conference, 2006.

[21] J Q. Wang Multi criteria interval intuitionistic fuzzy decisionmaking approach with incomplete certain information. Control and Decision, 2006(11): 1253-1256.

[22] J Q. Wang, Survey on fuzzy multi-criteria decision making approach. Control and Decision, 2008(2).

[23] J P. Xu, Technique of order preference by similarity to ideal and anti-ideal alternative for multiple attribute group decision making problems based on Hausdorff metric. Systems Engineering Theory and Practice, 2002, 22(10): 84-93.

[24] Z S. Xu, Study on method for triangular fuzzy number based multi-attribute decision making with preference information on alternatives. Systems Engineering and Electronics, 2002, 24(8): 9-12.

[25] Z S. Xu, Da Q L. Possibility degree method for ranking interval numbers and its application. Journal of Systems Engineering, 2003(18): 67-70.

[26] L. A. Zadeh, Fuzzy Sets, Inf. Control, 8 (3), 338-353 :( 1965). 